\title{
Lien B. Luu, Immigrants and the Industries of London
} 1500-1700

Aldershot, Ashgate, 2005, 366 pages.

Liliane Hilaire-Pérez

\section{OpenEdition}

\section{Journals}

Édition électronique

URL : http://journals.openedition.org/dht/1118

DOI : $10.4000 /$ dht. 1118

ISSN : $1775-4194$

Éditeur :

Centre d'histoire des techniques et de l'environnement du Cnam (CDHTE-Cnam), Société des élèves du CDHTE-Cnam

\section{Édition imprimée}

Date de publication : 1 décembre 2007

Pagination : 145-148

ISBN : 978-2-9530779-0-2

ISSN : 0417-8726

Référence électronique

Liliane Hilaire-Pérez, « Lien B. Luu, Immigrants and the Industries of London 1500-1700 », Documents pour I'histoire des techniques [En ligne], 14 | $2^{\mathrm{e}}$ semestre 2007, mis en ligne le 03 novembre 2010, consulté le 24 septembre 2020. URL : http://journals.openedition.org/dht/1118; DOI : https://doi.org/10.4000/ dht. 1118

Ce document a été généré automatiquement le 24 septembre 2020

(C) Tous droits réservés 


\section{Lien B. Luu, Immigrants and the Industries of London 1500-1700}

Aldershot, Ashgate, 2005, 366 pages.

Liliane Hilaire-Pérez

\section{RÉFÉRENCE}

Lien B. Luu, Immigrants and the Industries of London 1500-1700, Aldershot, Ashgate, 2005, 366 pages.

1 Le livre de Lien B. Luu renouvelle l'histoire des migrations dans le monde du travail à Londres aux XVe et XVIe siècles. Analysant une période où les savoirs techniques sont peu codifiés, où l'acquisition des techniques nouvelles est indissociable des mobilités humaines, l'auteur étudie les modalités de transmission des savoirs tout en évitant les écueils diffusionnistes et les stéréotypes sur le rôle déterminant de telle communauté, de telle minorité, auxquelles ont assignerait des identités professionnelles et des compétences figées. Loin des clichés, Lien B. Luu souligne, dès le début du livre, la fluidité des identités au travail, les migrants s'adaptant aux activités des communautés étrangères installées et aux contraintes imposées par les autorités. On perçoit l'originalité du propos de l'auteur: étudier les étrangers, non seulement dans leurs caractéristiques propres, mais aussi à la lumière de leur réception par les natifs et par leurs compatriotes déjà sur place.

Inadéquation des qualifications (mismatch), hétérogénéité des communautés, circuits complexes avec persistance des retours au pays d'origine, pluralité des migrations (en réseaux ou chain migration, circulaires, en masse), différenciation des modèles de diffusion selon les métiers, selon les périodes, ambiguïté des politiques migratoires : ces approches croisées permettent d'éviter tout cliché, au profit d'une réflexion argumentée, contextualisée et ouverte aux débats.

3 Cette analyse fine est rendue possible par une enquête archivistique d'ampleur dans les dénombrements gouvernementaux (certificates ou returns of aliens), dans les fonds des 
corporations de Londres (brasseurs, orfèvres, tisserands), dans ceux de la municipalité de Londres et des Églises étrangères (stranger churches). S'y ajoutent des recherches dans les archives des pays d'origine, notamment dans l'actuelle Belgique (archives du Conseil des troubles à Bruxelles, fonds municipaux à Anvers).

4 L'auteur a établi des fichiers de milliers d'individus, d'une immense richesse, qu'elle restitue sous forme de nombreux tableaux, dans le corps du texte et en annexe. Ces données chiffrées constituent une véritable innovation dans l'étude de ces migrations. Elles permettent à l'auteur de confronter les discours, les représentations et les législations à la réalité du nombre, des origines, des métiers, des fortunes et des appartenances religieuses.

5 Cette saisie globale du fait migratoire structure les premiers chapitres du livre. Après une brillante description de l'essor des marchés de consommation dans la capitale, l'auteur étudie les politiques d'encouragement à l'immigration au XVIe siècle. Elle reprend ainsi la question des patents et de leurs liens avec la gestion des migrations, en revenant sur le rôle pionnier du chancelier William Cecil sous le règne d'Elisabeth (1558-1603). Un aspect important est développé, l'obligation faite aux patentés d'enseigner leurs techniques aux natifs (à un moment où est promulgué le Statut des apprentis, 1563). Il est toujours utile de rappeler que l'investissement de l'État dans l'innovation ne fut pas l'apanage du continent.

6 Londres, cité en expansion forte d'une consommation en plein essor, ville favorisée par les mesures incitatives du gouvernement et par la tolérance religieuse, attire les étrangers. Dans le chapitre IV, Lien B. Luu confronte les différents dénombrements livrés par les historiens et propose le sien : jusqu'à 10000 étrangers à Londres en 1571, soit $10 \%$ de la population et trois phases migratoires, rythmées par les persécutions religieuses sur le continent, par la reconquête des Pays-Bas méridionaux (1585) qui signe le point de non-retour pour bien des émigrés, et par les ambiguïtés des mesures légales anglaises, favorisant tout en les limitant les entrées des étrangers. À mesure, le profil des migrants évolue: Hollandais du nord puis Flamands, artisans de métiers traditionnels puis de métiers du luxe innovateurs (soie, dentelle, orfèvrerie, sucrerie, verrerie, amidonnerie). Ce chapitre, dense, évoque aussi l'installation des étrangers dans la métropole. Le principal moteur de la répartition professionnelle des migrants à Londres est la demande londonienne et les opportunités qu'elle leur offre. Les métiers du luxe sont en première ligne. Ils sont aussi moins rétifs à l'accueil des étrangers que ceux régis par d'anciennes corporations (tailleurs, cordonniers). Les conditions d'accueil déterminent également pour une large part la distribution géographique : la politique municipale et gouvernementale de dispersion des migrants limite leur concentration le long de la Tamise, les incite à s'installer aux limites de la Cité (Bishopgate) et dans les enclaves privilégiées, les liberties, où vivent aussi les foreigners, étrangers à la ville, et les artisans non corporés. La ségrégation spatiale est avant tout le résultat d'une politique migratoire, même si l'immigration en réseau, chain migration, renforce les implantations de départ.

7 L'intégration juridique est la clé de voûte des conditions faites aux migrants et des perceptions développées à leur encontre. C'est le sujet du chapitre V où Lien B. Luu offre une synthèse réussie des statuts des étrangers à Londres au XVIe siècle. Aux contraintes édictées envers les aliens depuis Richard III, qui portent sur les droits de propriété plus que sur les conditions d'emploi, s'ajoutent le statut de denizen, sujet d'adoption, les naturalisations, l'acquisition du droit de bourgeoisie (freedom) qui 
distingue le freeman du foreigner et dépend de l'apprentissage (réservé aux natifs), les rédemptions, achats et autres privilèges dérogatoires. De façon judicieuse, l'auteur montre que l'appartenance à ces différents groupes influe sur l'attitude des natifs à l'égard des étrangers. La poussée xénophobe après 1580 , sujet de débat entre historiens, est favorisée par la visibilité accrue des étrangers, par leur stigmatisation légale, à un moment de difficulté économique et de pression démographique urbaine. Les tensions suscitent d'une part, des retours au pays d'origine et d'autre part, des mesures gouvernementales (dispersion, enseignement, emploi de natifs) pour répondre aux accusations de repli sur soi des étrangers, de secret et de concurrence pour l'emploi. La City se montre plus vindicative, restreignant l'immigration aux seuls protestants persécutés, entravant l'immigration économique, excluant les enfants d'étrangers de l'apprentissage chez les natifs, non sans susciter des controverses sur la définition de nationalité.

8 Posé ce cadre général, Lien B. Luu étudie trois cas particuliers, la soierie, l'orfèvrerie et la brasserie. Elle met ainsi en valeur la forte différenciation des conditions faites aux étrangers selon les secteurs, les corporations et les origines géographiques. Trois constantes cependant se dessinent : la fluidité des identités professionnelles, l'intensité des transmissions de savoirs à l'intérieur des communautés de migrants et la tendance des autorités à limiter, filtrer, contrôler l'immigration économique, une politique qui, à terme, restreint les bénéfices que l'Angleterre pouvait espérer de la main-d'œuvre hollandaise et flamande. Le facteur clé des circulations techniques, dans tous les cas, réside dans l'organisation du marché du travail.

9 La soierie est emblématique de la flexibilité des compétences professionnelles. Si ce secteur est introduit en Angleterre au XVe siècle par des Wallons, de Bruges et d'Anvers, les migrants du siècle suivant, des Wallons protestants persécutés, n'appartiennent pas nécessairement à la soierie ou bien, ils ne sont pas tisserands. C'est à Londres qu'ils s'adaptent aux activités de la communauté, profitant des opportunités offertes et acquérant des compétences pour le tissage. Loin de tout modèle diffusionniste binaire d'importation et de dissémination des savoirs, l'auteur explique que les compétences sont d'abord diffusées dans le groupe, parmi les migrants, ce que renforce la concentration géographique (Bishopgate, puis Spitalfields). La corporation des tisserands réagit par l'intégration des plus qualifiés, des plus inventifs (avec examen de tissus nouveaux), par la fermeture aux migrants économiques et par une politique active d'apprentissage en faveur des natifs. Les étrangers sont tenus d'employer des apprentis et des compagnons anglais dans le tissage et non seulement dans les tâches requérant moins de qualification. La distinction entre formal training et pauper apprenticeship est capitale, suggérant que les étrangers ont pu fournir des emplois sans transmettre de compétences, suscitant la vindicte des autorités locales. La diffusion des compétences repose sur une politique volontariste des autorités qui tentent de réguler le marché du travail. À terme, dans un troisième temps, la soierie devient un secteur d'activité londonien, investi par les natifs.

10 Périodisation fine, analyse conjuguée de la diffusion de savoirs, des politiques migratoires et du marché du travail : ces approches permettent aussi de saisir sous un nouveau jour l'impact des Huguenots dans l'orfèvrerie londonienne. Depuis le MoyenÂge, des étrangers hautement qualifiés dominent ce secteur mais leur présence est discontinue, ne permettant pas de former une main-d'œuvre qualifiée permanente. Ce sont les migrations circulaires de Flamands, wanderjahre (tour d'Europe des 
compagnons), puis l'installation temporaire de compagnons hollandais et allemands, employés dans des réseaux de sous-traitance comme main-d'œuvre flexible. La situation change au XVIIe siècle avec l'installation définitive de Français persécutés, mieux acceptés par les autorités. Ils acquièrent la citoyenneté (freedom), entrent dans la corporation, forment la deuxième génération tout en transmettant leurs savoirs aux natifs. La greffe des compétences passe par le travail d'apprentis anglais chez des étrangers grâce à des aides publiques. L'impact des Huguenots découle de cet accueil favorable. Sur un pied d'égalité avec les orfèvres anglais, ils sont des concurrents sérieux et élèvent ainsi le niveau de compétence général, suscitant des sentiments contradictoires chez les natifs.

11 La brasserie offre un autre cas de figure, plus marqué par le poids des facteurs économiques. L'introduction de la bière par les Hollandais à Londres au XVe siècle, pour répondre à la demande des Doche de la City (Hollandais, Allemands, Flamands), se heurte à l'opposition des producteurs d'ale, breuvage à base de malt, d'eau et de levure. La suspicion à l'égard de la bière, considérée comme moins nutritive et même assimilée à un poison, se mêle aux craintes pour l'emploi, la fabrication de la bière requérant des savoir-faire plus importants et excluant ainsi les brasseurs d'ale de la production de bière dont le succès va croissant. Le soutien politique est déterminant, aboutissant à la création d'une corporation qui finit par fusionner avec celle de brasseurs d'ale au début $\mathrm{du}$ XVIe siècle. Ce regroupement inaugure pourtant la domination du secteur par les Anglais et une discrimination envers les étrangers. Dans un premier temps, les compétences se diffusent via le marché de l'emploi (étrangers au travail chez des brasseurs anglais). Puis l'augmentation de la demande favorisant une concentration de la production, donc une division du capital et du travail, l'élévation du seuil d'entrée dans le métier profite aux Anglais, mieux armés que les jeunes immigrés pour financer et pour gérer ces entreprises complexes. L'anglicisation de la brasserie est acquise dans les années 1650. Le changement de structure de la production explique le recul des étrangers dans la profession.

Ces trois exemples, au-delà de leurs particularités, suggèrent la nécessité de périodiser, de distinguer des phases dans les processus de diffusion. Comme le démontre Lien B. Luu, les circulations techniques ne revêtent pas la même intensité dans le temps et dans l'espace et c'est la tâche des historiens de comprendre les accélérations, les ralentissements et les échecs, les temporalités différenciées, loin de toute success story héroïque du progrès technique.

En guise de synthèse, Lien $B$. Luu propose une typologie des migrants, des migrations et des modalités de transmission des compétences, mettant l'accent sur le marché du travail, sur l'emploi des natifs par les étrangers et suggérant que les migrants ont contribué à créer des emplois pour les Anglais, contrairement aux discours xénophobes. Ceux-ci se développent à la faveur d'une visibilité accrue des étrangers, stigmatisés par une législation ambiguë. L'auteur conclut au rôle bénéfique des immigrés pour le développement anglais, rejoignant ainsi les analyses récentes sur l'importance du creuset européen qui a présidé à la révolution industrielle anglaise. L'immigration (plus que le charbon) apparaît comme la clé de voûte de la croissance anglaise.

14 Des annexes, un inventaire des sources, une bibliographie dense, un index des noms propres et thématique ajoutent à la qualité de cet ouvrage, aussi important pour 
l'histoire de Londres que pour celle des mobilités, des constructions identitaires et de la transmission des savoirs techniques.

\section{AUTEURS}

LILIANE HILAIRE-PÉREZ

CDHTE-Cnam 